\title{
SiO maser survey of AGB stars in the North Galactic Cap ${ }^{\star}$
}

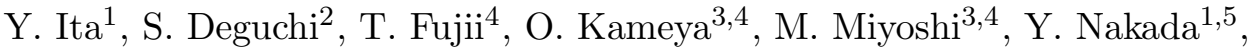 \\ J. Nakashima ${ }^{6}$, and M. Parthasarathy ${ }^{7,8}$
}

1 Institute of Astronomy, School of Science, The University of Tokyo, 2-21-1 Osawa, Mitaka, Tokyo 181-0015, Japan

2 Nobeyama Radio Observatory, National Astronomical Observatory, Minamimaki, Minamisaku, Nagano 384-1305, Japan

3 Mizusawa Astrogeodynamics Observatory, National Astronomical Observatory, Mizusawa, Iwate 023-0861, Japan

4 VERA project office, National Astronomical Observatory, Mitaka, Tokyo 181-8588, Japan

${ }^{5}$ Kiso Observatory, School of Science, The University of Tokyo, Mitake, Kiso, Nagano 397-0101, Japan

6 Department of Astronomical Science, The Graduate University for Advanced Studies, Nobeyama Radio Observatory, National Astronomical Observatory, Minamimaki, Minamisaku, Nagano 384-1305, Japan

7 National Astronomical Observatory, 2-21-1 Osawa, Mitaka, Tokyo 181-8588, Japan

8 Indian Institute of Astrophysics, Bangalore 560034, India

Received 4 December 2000 / Accepted 1 June 2001

\begin{abstract}
A SiO maser survey in the $J=1-0, v=1$ and 2 transitions has been made for IRAS sources in the North Galactic Cap $\left(b>30^{\circ}\right)$ with the Nobeyama $45 \mathrm{~m}$ radio telescope. The sources were selected on the basis of their IRAS $12 / 25-\mu \mathrm{m}$ and $25 / 60-\mu \mathrm{m}$ flux ratios as likely oxygen-rich AGB candidates. SiO masers were detected from 24 out of the 97 selected sources, where 17 were new detections. The distances and heights above the Galactic plane are calculated. The Galactic distribution of detected and undetected stars indicates that metallicity is likely to govern the detection rate. The Galactocentric angular velocities of the subsampled stars are derived and their variation with the Galactic height is discussed.
\end{abstract}

Key words. late-type star - radio lines - galactic kinematics and structure

\section{Introduction}

$\mathrm{SiO}$ masers have been detected from late-type stars of considerably different types of variability and wide range of mass-loss rates. The widespread occurrence of $\mathrm{SiO}$ masers among different classes of Asymptotic Giant Branch (AGB) stars, including those with very low massloss rates (e.g., $\sim 10^{-8} M_{\odot} \mathrm{yr}^{-1}$ for $\mathrm{R}$ Leo, Lane et al. 1987), indicates that unlike $\mathrm{OH}$ and $\mathrm{H}_{2} \mathrm{O}$, $\mathrm{SiO}$ masers are likely to arise from the innermost regions of their circumstellar envelopes. Recent interferometic observations show that $\mathrm{SiO}$ masers are indeed located very close to the central star (e.g., Cohen 1989; Miyoshi et al. 1994). Also, the pumping mechanism for $\mathrm{SiO}$ masers proposed by

Send offprint requests to: Y. Ita,

e-mail: yita@mtk.ioa.s.u-tokyo.ac.jp

* Based on observations at the Nobeyama Radio Observatory (NRO). NRO is a branch of the National Astronomical Observatory, an inter-university research institute, operated by the Ministry of Education, Science and Culture, Japan.
Deguchi \& Iguchi (1976) predicts that the maser beam is directed tangentially to the radial outflows. This implies that the central velocity of the $\mathrm{SiO}$ maser line equals the line of sight (l.o.s.) velocity of the star within a few $\mathrm{km} \mathrm{s}^{-1}$ accuracy (Jewell et al. 1991; Jiang et al. 1995). Therefore, it is a powerful tool for investigating stellar radial velocities.

Up to now, $\mathrm{SiO}$ maser surveys have been made, for example, toward the Galactic Bulge (Nakada et al. 1993; Izumiura et al. 1994, 1995a, 1995b), toward the outer disk of the Galaxy (Jiang et al. 1996). In spite of the many $\mathrm{SiO}$ maser observations, little attention has been paid to stars in the North Galactic Cap. This region is very interesting because one has an easier access to the thick disk population and can investigate the nature of its constituent stars.

In this paper, we present the result of the $\mathrm{SiO}$ maser survey of the AGB stars in the North Galactic Cap. Based on this result, we discuss the spatial distribution of the high galactic latitude maser sources. Also, based on the 
l.o.s. velocities, we discuss the vertical kinematics of the Galaxy.

\section{Source selection}

The IRAS Point Source Catalog (IRAS PSC) (IRAS Science Team 1988) is at present still the most comprehensive catalog of IR sources, although more comprehensive all sky NIR survey data, e.g., the Two Micron All Sky Survey, is recently getting available in part. Therefore, stars which satisfy all of the four following criteria

1. $b>30^{\circ}$;

2. $f_{12}>3(\mathrm{Jy})$

3. $-0.301<C_{12}\left(\equiv \log \left[f_{25} / f_{12}\right]\right)<0.6$;

4. $C_{23}\left(\equiv \log \left[f_{60} / f_{25}\right]\right)<-0.48$,

were selected from version 2 of the IRAS PSC, where $b$ is the galactic latitude and $f_{\lambda}$ denotes the flux density in Jansky in wavelength $\lambda$ as detected by IRAS. As a result, the sample studied here consists of 97 IRAS sources.

In the direction away from the plane, there are many observational advantages; (1) one has reliable IRAS 60 and $100 \mu \mathrm{m}$ measurements, (2) interstellar extinction can be reasonably neglected, and (3) there is no point source confusion.

The selected stars are listed in Table 1, together with their IRAS flux density ratios. Also $(l, b)$ calculated from the IRAS position, IRAS LRS spectral class (LRS), the pulsation periods, and the $\mathrm{SiO}$ maser observation results are tabulated.

\section{Observation}

The $J=1-0, v=1$ and 2 maser lines of $\mathrm{SiO}$ at the rest frequencies of $43.122 \mathrm{GHz}$ and $42.821 \mathrm{GHz}$, respectively, were observed with the Nobeyama $45 \mathrm{~m}$ radio telescope during six sessions, 1998 May, June, 1999 June, and 2000 Apr., May, June. A cooled SIS receiver with a bandwidth of about $0.5 \mathrm{GHz}$ was used. The aperture efficiency of the telescope was about 0.61 at $43 \mathrm{GHz}$. The half-power beam width (HPBW) was about $40^{\prime \prime}$ at $43 \mathrm{GHz}$.

An acousto-optical spectrometer array of a high resolution (AOS-H) was used. Each spectrometer has a $40 \mathrm{MHz}$ bandwidth and 2048 frequency channels, giving the velocity coverage of about $280 \mathrm{~km} \mathrm{~s}^{-1}$ and the spectral resolution of $0.28 \mathrm{~km} \mathrm{~s}^{-1}$ (per two binned channels). For fear of missing high-velocity $\left(\left|V_{\mathrm{LSR}}\right|>200 \mathrm{~km} \mathrm{~s}^{-1}\right)$ components, a low resolution (AOS-W) array was also used. This has the velocity coverage of about $1700 \mathrm{~km} \mathrm{~s}^{-1}$ and the spectral resolution of $1.7 \mathrm{~km} \mathrm{~s}^{-1}$ (per two binned channels).

Observations were made in a position switching mode, where the off-position was chosen 5 arcmin away from the on-position in azimuth. The antenna temperature of the detection limit was $0.2 \mathrm{~K}$ at the $4 \sigma$ level. The system temperature (including atmospheric noise) was normally around 200 to $300 \mathrm{~K}$, depending mainly on the weather condition and elevation angle of the antenna. The conversion factor from antenna temperature to flux density was $2.9 \mathrm{Jy} \mathrm{K}^{-1}$.
The observations were made on the IRAS positions. Regular pointing checks and data calibration were made using the strong $\mathrm{SiO}$ maser sources, e.g., R LMi, U Her and W Hya etc. The pointing accuracy was usually found to be better than 10 arcsec.

We judged the detection of the $\mathrm{SiO}$ masers by the following criteria.

1. For a narrow spike-type emission, the peak antenna temperature must be greater than $10 \sigma$ and the line width greater than 6 channels $\left(1.0 \mathrm{~km} \mathrm{~s}^{-1}\right)$;

2. For broad emission, $S / N$ must be larger than 10; the effective $S / N$ is calculated from the half-power line width (the integrated intensity divided by half the peak intensity) and the average rms noise (corrected for the half-power line width).

\section{Results and discussion}

\subsection{SiO maser detection rate}

The $\mathrm{SiO}$ maser spectra of detected sources are displayed in Fig. 1. The observational results are summarized in Table 2 for detected sources and in Table 3 for nondetected sources. In Table 2, velocity in Local Standard of Rest frame (LSR), antenna temperature, the integrated flux, rms of the noise level and the date of observation in the format, YYMMDD.D are tabulated. In Table 3, rms of the noise level for each transition and observation date are tabulated. Stars marked "a" on the right shoulder of their IRAS name do not satisfy our selection criteria, but observed in the extra time, and are excluded from the later analysis.

Of the 97 sources in the North Galactic Cap, 16 had already been observed previously and had given 7 detections. Of the 83 sources observed in this survey, 17 detections are new. In total, $\mathrm{SiO}$ masers are detected in 24 out of the 97 sources, and the detection rate is about $25 \%$. This value is much less than that of the previous surveys, $56 \%$ toward the near end of the Galactic bar (Izumiura et al. 1999), $62 \%$ toward the bulge (Jiang et al. 1995; Izumiura et al. 1994, 1995a, 1995b) and even less than 35\% toward the outer disk (Jiang et al. 1996) even thouth the very similar color selection criteria and the same telescope was used.

This lower detection rate is partly attributed to the decrease of the number of oxygen-rich long-period Mira variables in the direction away from the Galactic plane. Distribution studies in the Galaxy suggest that the intermediate-period $\left(300^{\mathrm{d}}<P<400^{\mathrm{d}}\right)$ oxygen-rich Miras, that are most likely to emit $\mathrm{SiO}$ maser radiation, belong to the thin disk population (Jura \& Kleinmann 1992a, 1992b; Kerschbaum \& Hron 1992). On the other hand, shortperiod $\left(P<300^{\mathrm{d}}\right)$ oxygen-rich Miras or Semi-regular variables, that are on average farther from the Galactic plane and less likely to emit $\mathrm{SiO}$ masers, belong to the thick disk population (Jura 1994). 
Table 1. Data of the sampled stars.

\begin{tabular}{|c|c|c|c|c|c|c|c|c|c|}
\hline $\begin{array}{l}\text { IRAS } \\
\text { Name }\end{array}$ & $\begin{array}{c}l \\
(\mathrm{deg})\end{array}$ & $\begin{array}{c}b \\
(\mathrm{deg})\end{array}$ & $C_{12}$ & $C_{23}$ & $\overline{\mathrm{LRS}}$ & $\begin{array}{c}\mathrm{SiO}^{*} \\
(\mathrm{Y} / \mathrm{N})\end{array}$ & $\begin{array}{l}\text { Period } \\
\text { (days) }\end{array}$ & $\begin{array}{c}\text { Distance } \\
\text { (pc) }\end{array}$ & $\begin{array}{l}\text { GCVS } \\
\text { Name }\end{array}$ \\
\hline $08016+4107$ & 179.4 & 30.7 & -0.19 & -0.74 & 29 & $\mathrm{Y}$ & & 1678.1 & \\
\hline $08020+6637$ & 149.4 & 32.2 & -0.25 & -0.81 & & $\mathrm{~N}$ & & 1818.5 & \\
\hline $08134+4017$ & 180.8 & 32.8 & -0.18 & -0.76 & & $\mathrm{~N}$ & 295.2 & 3862.5 & W Lyn \\
\hline $08258+6237$ & 153.9 & 35.2 & -0.26 & -0.84 & & $\mathrm{~N}$ & & 2398.7 & \\
\hline $08430+3705$ & 185.8 & 38.0 & -0.04 & -1.05 & & $\mathrm{~N}$ & & 4080.5 & \\
\hline $09005+0824$ & 221.0 & 33.0 & -0.28 & -0.67 & & $\mathrm{~N}$ & & 1839.7 & \\
\hline $09057+1325$ & 216.2 & 36.3 & -0.30 & -0.75 & 15 & $\mathrm{~N}^{a}$ & & 504.0 & CW Cnc \\
\hline $09069+2527$ & 201.9 & 40.7 & -0.29 & -0.76 & 23 & $\mathrm{Y}^{a}$ & 393.2 & 721.4 & W Cnc \\
\hline $09276+4454$ & 175.5 & 46.6 & 0.18 & -0.85 & & $\mathrm{~N}^{d}$ & & 3245.5 & \\
\hline $09343-0536$ & 240.5 & 32.6 & -0.28 & -0.67 & & $\mathrm{~N}$ & & 2736.0 & \\
\hline $09406+5359$ & 161.9 & 46.7 & -0.26 & -1.02 & & $\mathrm{~N}$ & 326.0 & 1726.9 & YY UMa \\
\hline $09439-0547$ & 242.5 & 34.3 & -0.29 & -0.89 & & $\mathrm{~N}$ & & 1681.9 & TT Sex \\
\hline $10196+2545$ & 207.1 & 56.7 & -0.29 & -0.90 & & $\mathrm{~N}$ & & 957.3 & \\
\hline $10353-1145$ & 258.9 & 39.2 & -0.24 & -0.80 & & $\mathrm{~N}$ & 85.0 & 345.1 & FF Hya \\
\hline $10411+6902$ & 138.4 & 44.4 & -0.23 & -0.89 & 23 & $\mathrm{Y}^{a}$ & 301.6 & 624.8 & R UMa \\
\hline $10457+3633$ & 185.8 & 62.4 & -0.24 & -0.82 & 15 & $\mathrm{~N}$ & & 1386.3 & \\
\hline $10521+7208$ & 134.7 & 42.5 & -0.25 & -0.68 & 26 & Y & 215.2 & 792.2 & VX UMa \\
\hline $10594-0723$ & 261.7 & 46.3 & -0.20 & -0.81 & & $\mathrm{~N}$ & 342.7 & 2758.0 & RT Crt \\
\hline $11125+7524$ & 130.8 & 40.5 & -0.26 & -0.85 & 23 & Y & & 567.5 & CS Dra \\
\hline $11193-2443$ & 278.4 & 33.6 & -0.24 & -0.83 & & $\mathrm{~N}$ & & 1158.0 & \\
\hline $11252+1525$ & 240.4 & 67.2 & -0.19 & -0.90 & & $\mathrm{~N}$ & 107.0 & 544.5 & AF Leo \\
\hline $11269-1839$ & 277.4 & 39.8 & -0.30 & -0.61 & & $\mathrm{~N}$ & & 2621.5 & \\
\hline $11443+5830$ & 138.4 & 56.9 & -0.24 & -0.99 & & $\mathrm{~N}$ & & 1813.5 & WW UMa \\
\hline $11445+4344$ & 157.2 & 69.1 & -0.15 & -0.90 & & $\mathrm{~N}$ & & 917.8 & AZ UMa \\
\hline $11472-0800$ & 277.9 & 51.6 & 0.10 & -0.90 & & $\mathrm{~N}^{d}$ & & 4239.8 & \\
\hline $11567-2652$ & 288.9 & 34.3 & -0.20 & -0.94 & 29 & $\mathrm{Y}$ & & 1830.6 & \\
\hline $12016+1903$ & 248.0 & 76.3 & -0.28 & -0.68 & 24 & $\mathrm{Y}^{a}$ & 362.8 & 1263.0 & R Com \\
\hline $12020+0254$ & 276.4 & 63.0 & -0.22 & -0.77 & 26 & $\mathrm{~N}$ & 134.0 & 706.5 & TZ Vir \\
\hline $12120-0545$ & 286.5 & 55.7 & -0.24 & -0.73 & & $\mathrm{Y}^{b}$ & 339.5 & 1721.5 & T Vir \\
\hline $12219-0445$ & 290.5 & 57.2 & -0.28 & -0.68 & & $\mathrm{~N}$ & & 1737.6 & \\
\hline $12254+1904$ & 268.7 & 80.1 & -0.27 & -0.95 & & $\mathrm{~N}$ & & 1897.3 & TV Com \\
\hline $12323-2033$ & 297.7 & 41.9 & -0.28 & -0.76 & 14 & $\mathrm{~N}$ & & 1227.2 & RU Crv \\
\hline $12326-2017$ & 297.8 & 42.1 & -0.29 & -0.64 & & $\mathrm{~N}$ & & 2220.0 & \\
\hline $12344+2720$ & 213.2 & 86.8 & -0.30 & -0.87 & & $\mathrm{~N}^{a}$ & & 553.5 & \\
\hline $12449+3838$ & 127.1 & 78.7 & -0.18 & -0.87 & 26 & $\mathrm{~N}^{a}$ & 345.6 & 1677.2 & $\mathrm{U} C \mathrm{C} n$ \\
\hline $12462+0738$ & 301.0 & 70.2 & -0.29 & -0.63 & & $\mathrm{~N}$ & & 2654.5 & \\
\hline $12562+2324$ & 325.6 & 85.7 & -0.23 & -0.73 & 28 & $\mathrm{~N}^{a}$ & 406.0 & 1253.6 & T Com \\
\hline $12564-3125$ & 304.8 & 31.1 & -0.28 & -0.66 & & $\mathrm{~N}$ & & 2611.6 & \\
\hline $13006-2850$ & 306.1 & 33.7 & -0.22 & -0.77 & & $\mathrm{~N}$ & 218.8 & 2143.6 & SW Hya \\
\hline $13053-3208$ & 307.0 & 30.3 & -0.27 & -0.67 & & $\mathrm{~N}$ & & 1953.0 & \\
\hline $13245-2446$ & 313.1 & 37.1 & -0.26 & -0.85 & 29 & $\mathrm{~N}$ & & 1657.8 & \\
\hline $13252-0254$ & 320.5 & 58.5 & -0.27 & -0.68 & & $\mathrm{~N}$ & 250.1 & 2489.7 & V Vir \\
\hline $13326-2207$ & 316.1 & 39.4 & -0.28 & -0.62 & & $\mathrm{~N}$ & & 2852.7 & \\
\hline $13367-3031$ & 315.0 & 31.0 & -0.24 & -0.64 & & $\mathrm{~N}$ & 253.0 & 2954.1 & V0603 Cen \\
\hline $13562-1342$ & 326.8 & 45.8 & -0.27 & -0.62 & 14 & $\mathrm{~N}$ & & 1397.8 & \\
\hline $13573+2801$ & 39.4 & 74.9 & -0.23 & -0.78 & 22 & $\mathrm{~N}$ & 120.0 & 765.0 & WY Boo \\
\hline $13582+3806$ & 73.8 & 72.0 & -0.19 & -0.77 & & $\mathrm{~N}$ & & 1049.8 & CY CVn \\
\hline $13594-0522$ & 333.1 & 53.1 & -0.29 & -0.63 & & $\mathrm{~N}$ & 313.0 & 2851.6 & AB Vir \\
\hline $14021-1909$ & 325.8 & 40.2 & -0.25 & -0.72 & & $\mathrm{~N}$ & & 2804.7 & \\
\hline $14086-0730$ & 334.8 & 50.1 & -0.13 & -0.78 & & $\mathrm{Y}^{c}$ & & 707.0 & IO Vir \\
\hline $14086-2839$ & 323.4 & 30.8 & -0.30 & -0.74 & 26 & $\mathrm{~N}^{a}$ & 331.5 & 584.6 & RU Hya \\
\hline $14142-1612$ & 330.9 & 41.7 & -0.27 & -0.77 & 22 & $\mathrm{~N}$ & & 666.4 & EW Vir \\
\hline $14247+0454$ & 352.7 & 58.0 & -0.22 & -0.74 & 24 & $\mathrm{Y}^{a}$ & 354.0 & 669.7 & RS Vir \\
\hline $14257-0530$ & 342.0 & 49.6 & 0.09 & -0.76 & & $\mathrm{~N}$ & & 7408.0 & \\
\hline $14303-1042$ & 339.2 & 44.7 & -0.20 & -0.82 & & $\mathrm{Y}$ & & 2350.2 & \\
\hline $14371+3245$ & 52.5 & 66.1 & -0.25 & -0.81 & 22 & $\mathrm{~N}^{a}$ & 137.0 & 319.1 & RV Boo \\
\hline $14382-2504$ & 332.1 & 31.3 & -0.23 & -0.81 & & $\mathrm{~N}$ & & 2127.4 & \\
\hline
\end{tabular}


Table 1. continued.

\begin{tabular}{|c|c|c|c|c|c|c|c|c|c|}
\hline $\begin{array}{l}\text { IRAS } \\
\text { Name }\end{array}$ & $\begin{array}{c}l \\
(\mathrm{deg})\end{array}$ & $\begin{array}{c}b \\
(\mathrm{deg})\end{array}$ & $\overline{C_{12}}$ & $C_{23}$ & $\overline{\mathrm{LRS}}$ & $\begin{array}{l}\mathrm{SiO}^{*} \\
(\mathrm{Y} / \mathrm{N})\end{array}$ & $\begin{array}{l}\text { Period } \\
\text { (days) }\end{array}$ & $\begin{array}{c}\text { Distance } \\
\text { (pc) }\end{array}$ & $\begin{array}{l}\text { GCVS } \\
\text { Name }\end{array}$ \\
\hline $14436-0703$ & 346.0 & 45.8 & -0.15 & -0.77 & & $\mathrm{~N}$ & & 4250.2 & \\
\hline $14524-2148$ & 337.4 & 32.5 & -0.29 & -0.73 & 24 & $\mathrm{Y}$ & 365.0 & 1280.8 & EG Lib \\
\hline $14534-0359$ & 351.6 & 46.6 & -0.29 & -0.71 & & $\mathrm{~N}$ & & 2527.4 & \\
\hline $14571-2135$ & 338.6 & 32.0 & -0.26 & -0.76 & 29 & $\mathrm{Y}$ & & 1821.5 & \\
\hline $15060+0947$ & 11.0 & 53.3 & -0.12 & -0.82 & 28 & Y & & 1475.6 & \\
\hline $15090-0549$ & 353.8 & 42.6 & -0.29 & -0.81 & & $\mathrm{Y}$ & 275.7 & 1389.5 & Y Lib \\
\hline $15106-1532$ & 346.0 & 35.0 & 0.16 & -0.64 & & $\mathrm{~N}$ & & 6178.7 & \\
\hline $15114-0142$ & 358.5 & 45.1 & -0.26 & -1.00 & 28 & $\mathrm{~N}$ & 432.7 & 1497.6 & Y Ser \\
\hline $15193+3132$ & 49.5 & 57.2 & -0.20 & -0.81 & 24 & $\mathrm{Y}^{a}$ & 360.3 & 528.1 & $\mathrm{~S} \mathrm{CrB}$ \\
\hline $15194-1829$ & 345.7 & 31.4 & -0.28 & -0.71 & 14 & $\mathrm{~N}$ & & 1356.6 & \\
\hline $15223-0203$ & 0.6 & 42.8 & -0.18 & -0.76 & 24 & $\mathrm{~N}$ & & 706.7 & OV Ser \\
\hline $15250+2952$ & 46.6 & 55.8 & -0.27 & -0.61 & & $\mathrm{~N}$ & & 3020.3 & \\
\hline $15255+1944$ & 29.5 & 53.5 & -0.19 & -0.91 & 29 & $\mathrm{Y}^{a}$ & 425.1 & 559.5 & WX Ser \\
\hline $15262+0400$ & 8.1 & 45.8 & -0.17 & -0.82 & 27 & $\mathrm{~N}^{a}$ & & 1075.9 & MW Ser \\
\hline $15334+2555$ & 40.3 & 53.4 & -0.25 & -0.96 & 28 & $\mathrm{~N}$ & 436.0 & 2176.6 & RU CrB \\
\hline $15367+1044$ & 18.4 & 47.3 & -0.29 & -0.81 & 29 & $\mathrm{~N}$ & & 1735.7 & \\
\hline $15415+0232$ & 9.6 & 41.9 & -0.23 & -0.73 & 14 & $\mathrm{~N}$ & & 1865.8 & \\
\hline $15517-1043$ & 358.6 & 31.6 & -0.20 & -0.65 & 14 & $\mathrm{~N}$ & & 1683.7 & GP Lib \\
\hline $15522-0749$ & 1.3 & 33.4 & -0.26 & -1.03 & & $\mathrm{~N}$ & & 1816.9 & \\
\hline $15540+0910$ & 19.4 & 42.8 & -0.11 & -0.36 & & $\mathrm{~N}$ & 280.0 & 3282.0 & RU Ser \\
\hline $15566+3609$ & 57.6 & 49.6 & -0.28 & -0.92 & 26 & $\mathrm{Y}$ & 332.2 & 763.0 & RS CrB \\
\hline $16030-0634$ & 4.5 & 32.1 & -0.26 & -0.69 & & $\mathrm{Y}$ & 340.4 & 2077.7 & BD Oph \\
\hline $16037+4218$ & 66.9 & 48.0 & -0.22 & -0.79 & 28 & Y & & 1787.3 & \\
\hline $16131-0216$ & 10.4 & 32.6 & -0.29 & -0.76 & 24 & $\mathrm{Y}$ & & 1213.8 & \\
\hline $16211+3057$ & 50.8 & 44.0 & -0.24 & -0.73 & 15 & $\mathrm{~N}$ & 90.0 & 575.1 & RY CrB \\
\hline $16241-0230$ & 11.9 & 30.2 & -0.22 & -0.93 & 28 & $\mathrm{~N}$ & & 1739.3 & V0707 Oph \\
\hline $16260+3454$ & 56.4 & 43.5 & -0.10 & -0.68 & 28 & $\mathrm{~N}^{a}$ & & 1176.7 & V0697 Her \\
\hline $16293+4900$ & 75.8 & 42.9 & -0.21 & -0.74 & & $\mathrm{~N}$ & & 2534.9 & \\
\hline $16330+0405$ & 19.9 & 31.9 & -0.24 & -0.74 & & $\mathrm{~N}$ & & 2754.0 & \\
\hline $16400+3301$ & 54.4 & 40.4 & -0.29 & -0.76 & 13 & $\mathrm{~N}$ & & 1417.3 & \\
\hline $16418+5435$ & 82.8 & 40.2 & -0.28 & -0.72 & & $\mathrm{~N}$ & & 2024.3 & \\
\hline $16418+5459$ & 83.3 & 40.2 & -0.25 & -0.78 & 24 & $\mathrm{~N}$ & 136.0 & 308.0 & S Dra \\
\hline $16560+2252$ & 43.1 & 34.5 & -0.24 & -0.72 & & $\mathrm{Y}$ & 222.0 & 2174.3 & MV Her \\
\hline $17050+1714$ & 37.7 & 30.5 & -0.22 & -0.77 & 14 & $\mathrm{Y}$ & 300.4 & 2330.7 & VY Her \\
\hline $17313+7033$ & 101.2 & 32.2 & -0.25 & -0.72 & & $\mathrm{~N}$ & & 2573.8 & \\
\hline $17329+5359$ & 81.6 & 32.8 & -0.21 & -0.72 & 25 & $\mathrm{Y}$ & 391.4 & 1297.8 & SY Dra \\
\hline $17343+7601$ & 107.5 & 31.0 & -0.28 & -0.90 & & $\mathrm{~N}$ & & 2369.4 & \\
\hline $17343+5026$ & 77.4 & 32.4 & -0.30 & -0.67 & & $\mathrm{~N}$ & 370.0 & 2426.8 & AO Her \\
\hline $17361+5746$ & 86.1 & 32.5 & -0.16 & -0.78 & 28 & $\mathrm{~N}$ & & 940.6 & TY Dra \\
\hline $17403+6234$ & 91.8 & 32.0 & -0.29 & -0.74 & 15 & $\mathrm{~N}$ & & 1033.6 & \\
\hline
\end{tabular}

Notes to Table 1:

$l, b$ : Galactic longitude and latitude calculated from the IRAS position.

* $\mathrm{SiO}$ : $\mathrm{SiO}$ maser detection results. Y means "detection" and $\mathrm{N}$ means "non-detection" respectively.

${ }^{a}$ Benson et al. (1990).

${ }^{b}$ Jewell et al. (1991).

${ }^{c}$ Hall et al. (1990) did not detect $\mathrm{SiO}$ maser from this star, but in this work, we detect.

${ }^{d}$ Fujii et al. in prep. (private communication).

\subsection{Detection vs. IRAS properties}

From the 45 stars in our sample with LRS spectra, 11 belong to class $1 \mathrm{n}$ and 34 to class $2 \mathrm{n}$. In those 45 stars, $\mathrm{SiO}$ emission was detected only from class $2 \mathrm{n}$ stars (17 out of 34 ), with one exception (IRAS $17050+1714$ ). It follows that the presence of $\mathrm{SiO}$ masers is strongly correlated with the LRS class $2 \mathrm{n}$, which indicates silicate emission at $10 \mu \mathrm{m}$.
In Fig. 2, we plotted both $\mathrm{SiO}$ maser detected and non-detected sources on the IRAS two-color diagram. The $\mathrm{SiO}$ maser detected stars are concentrated in a small area, the IIIa and VII regions as defined by Van der Veen \& Habing (1988). These areas are characterized by variable stars with circumstellar shells.

In the sample of 97 stars, there are only four with $C_{12}$ larger than $0.0(4 \%)$. This fraction is quite low in 

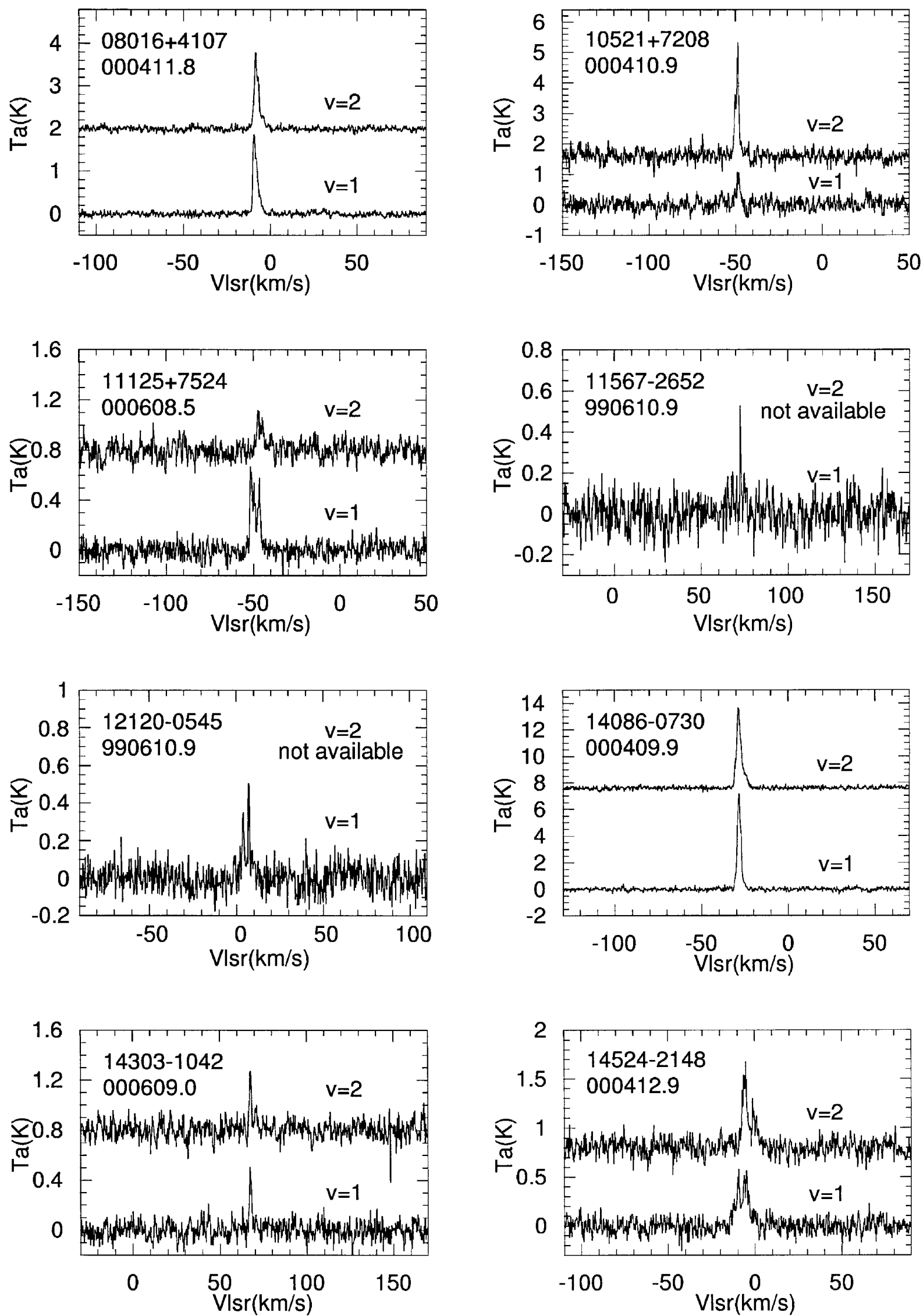

Fig. 1. Spectra of the $\mathrm{SiO} J=1-0, v=1$ and 2 lines for detected sources. 

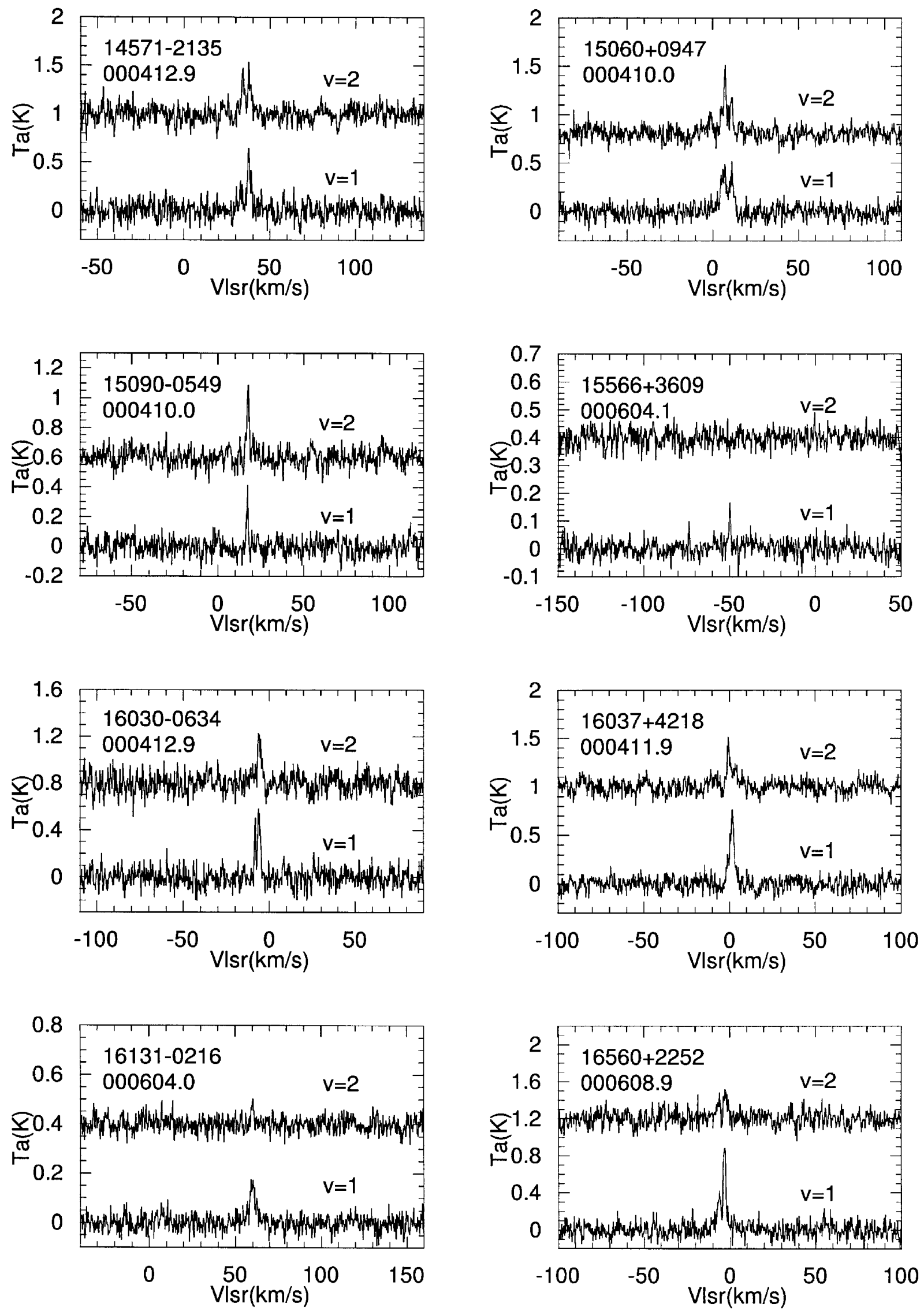

Fig. 1. continued. 

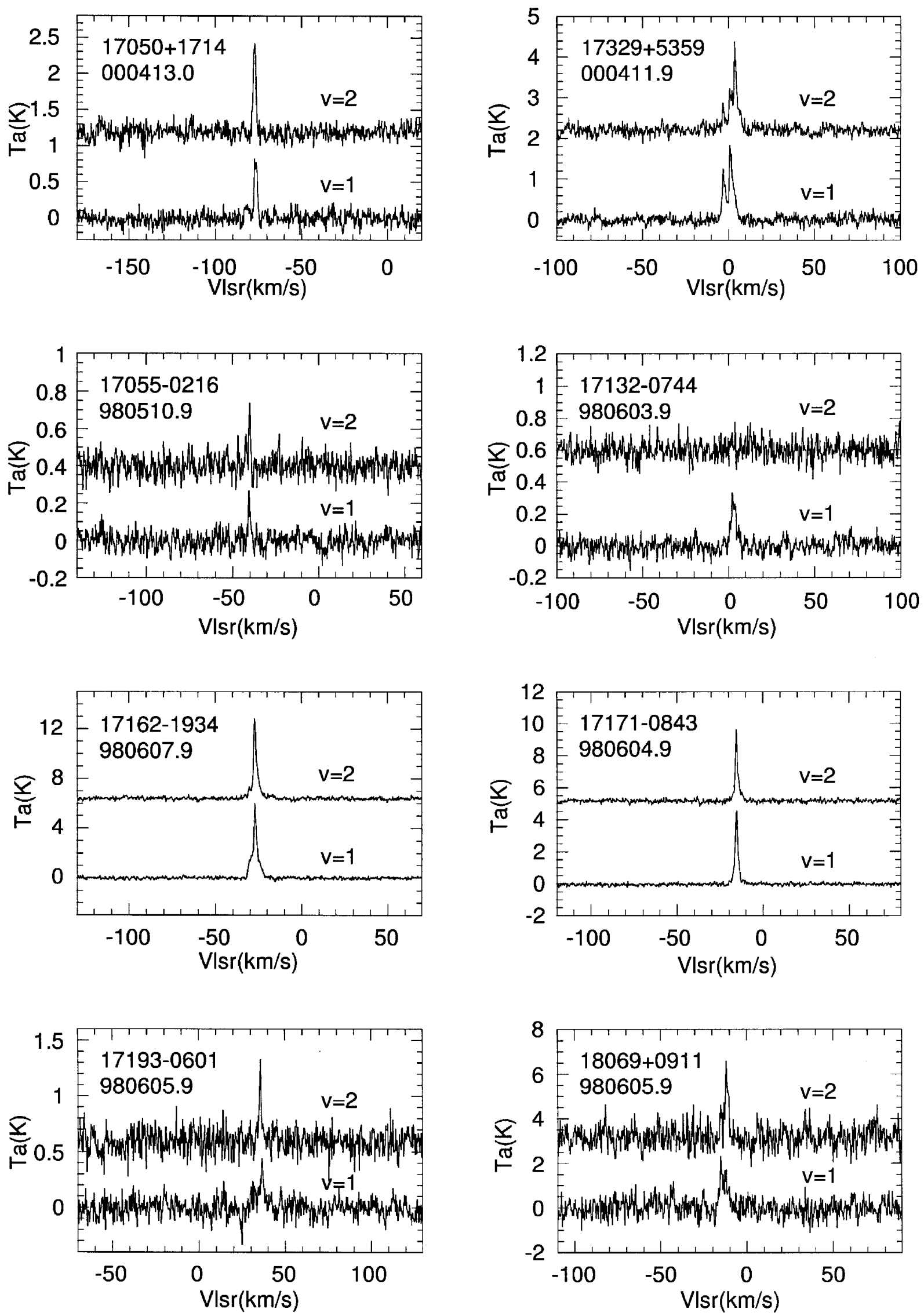

Fig. 1. continued. 

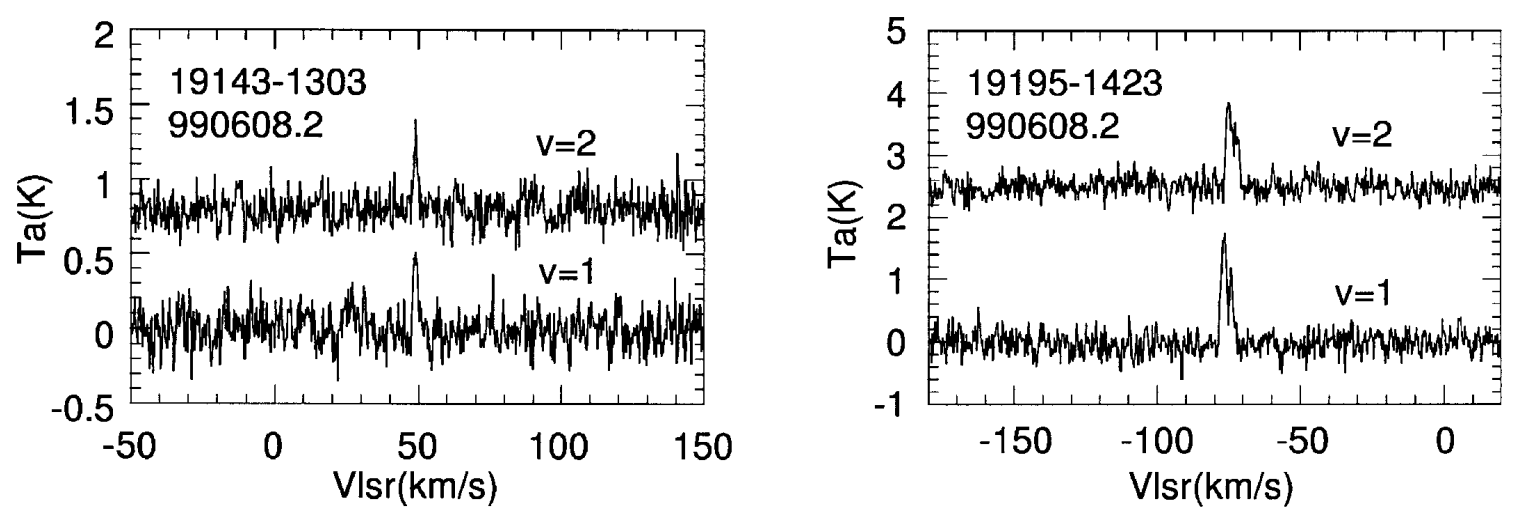

Fig. 1. continued.

Table 2. List of $\mathrm{SiO}$ maser detections.

\begin{tabular}{|c|c|c|c|c|c|c|c|c|c|}
\hline \multirow[b]{2}{*}{ IRAS Name } & \multicolumn{4}{|c|}{$\bar{J}=1-0, v=1$} & \multicolumn{4}{|c|}{$J=1-0, v=2$} & \multirow{2}{*}{$\begin{array}{c}\text { Observation } \\
\text { date } \\
\text { (yymmdd.d) }\end{array}$} \\
\hline & $\begin{array}{c}V_{\mathrm{lsr}} \\
\left(\mathrm{km} \mathrm{s}^{-1}\right)\end{array}$ & $\begin{array}{c}T_{\mathrm{a}} \\
(\mathrm{K})\end{array}$ & $\begin{array}{c}S \\
\left(\mathrm{~K} \mathrm{~km} \mathrm{~s}^{-1}\right)\end{array}$ & $\begin{array}{l}\mathrm{rms} \\
(\mathrm{K})\end{array}$ & $\begin{array}{c}V_{\text {lsr }} \\
\left(\mathrm{km} \mathrm{s}^{-1}\right)\end{array}$ & $\begin{array}{c}T_{\mathrm{a}} \\
(\mathrm{K})\end{array}$ & $\begin{array}{c}S \\
\left(\mathrm{~K} \mathrm{~km} \mathrm{~s}^{-1}\right)\end{array}$ & $\begin{array}{l}\mathrm{rms} \\
(\mathrm{K})\end{array}$ & \\
\hline $08016+4107$ & -9.3 & 1.875 & 5.899 & 0.047 & -8.6 & 1.814 & 5.769 & 0.048 & 000411.8 \\
\hline $10521+7208$ & -48.7 & 1.093 & 3.207 & 0.177 & -48.6 & 4.005 & 8.198 & 0.171 & 000410.9 \\
\hline $11125+7524$ & -51.7 & 0.668 & 2.640 & 0.058 & -47.0 & 0.362 & 1.134 & 0.062 & 000608.5 \\
\hline $11567-2652$ & 72.5 & 0.529 & 1.133 & 0.075 & - & - & - & - & 990610.9 \\
\hline $12120-0545$ & 7.2 & 0.530 & 1.458 & 0.064 & - & - & - & - & 990610.9 \\
\hline $14086-0730$ & -28.6 & 7.293 & 18.693 & 0.106 & -28.8 & 6.073 & 21.210 & 0.103 & 000409.9 \\
\hline $14303-1042$ & 67.5 & 0.507 & 1.112 & 0.062 & 67.7 & 0.492 & 1.192 & 0.065 & 000609.0 \\
\hline $14524-2148$ & -9.2 & 0.580 & 3.538 & 0.071 & -5.0 & 0.876 & 3.689 & 0.078 & 000412.9 \\
\hline $14571-2135$ & 37.6 & 0.644 & 2.134 & 0.083 & 37.6 & 0.536 & 1.961 & 0.080 & 000412.9 \\
\hline $15060+0947$ & 11.0 & 0.560 & 3.028 & 0.065 & 7.2 & 0.713 & 3.042 & 0.065 & 000410.0 \\
\hline $15090-0549$ & 17.2 & 0.413 & 0.134 & 0.056 & 17.6 & 0.488 & 0.958 & 0.055 & 000410.0 \\
\hline $15566+3609$ & -49.8 & 0.165 & 0.377 & 0.027 & -51.1 & 0.069 & -0.009 & 0.028 & 000604.1 \\
\hline $16030-0634$ & -6.0 & 0.582 & 1.412 & 0.072 & -6.4 & 0.443 & 1.164 & 0.082 & 000412.9 \\
\hline $16037+4218$ & 1.5 & 0.764 & 2.397 & 0.064 & -0.8 & 0.516 & 2.011 & 0.064 & 000411.9 \\
\hline $16131-0216$ & 60.3 & 0.182 & 0.769 & 0.029 & 60.2 & 0.102 & 0.187 & 0.029 & 000604.0 \\
\hline $16560+2252$ & -2.8 & 0.938 & 2.254 & 0.070 & -2.8 & 0.316 & 0.870 & 0.075 & 000608.9 \\
\hline $17050+1714$ & -76.6 & 0.839 & 2.228 & 0.081 & -77.2 & 1.215 & 2.134 & 0.080 & 000413.0 \\
\hline $17329+5359$ & 1.0 & 1.825 & 8.121 & 0.090 & 3.6 & 2.177 & 8.460 & 0.097 & 000411.9 \\
\hline $17055-0216^{a}$ & -40.6 & 0.268 & 0.204 & 0.044 & -40.1 & 0.379 & 0.310 & 0.052 & 980510.9 \\
\hline $17132-0744^{a}$ & 2.1 & 0.332 & 1.007 & 0.048 & $3.7 ?$ & 0.177 & 0.159 & 0.054 & 980603.9 \\
\hline $17162-1934^{a}$ & -27.2 & 6.140 & 19.528 & 0.086 & -27.4 & 6.424 & 18.532 & 0.103 & 980607.9 \\
\hline $17171-0843^{a}$ & -15.5 & 4.542 & 9.914 & 0.239 & -15.6 & 4.422 & 8.610 & 0.218 & 980604.9 \\
\hline $17193-0601^{a}$ & -36.5 & 0.437 & 1.415 & 0.081 & -35.8 & 0.728 & 1.292 & 0.100 & 980605.9 \\
\hline $18069+0911^{a}$ & -15.1 & 2.309 & 7.119 & 0.396 & -11.8 & 3.398 & 7.574 & 0.473 & 980605.9 \\
\hline $19143-1303^{a}$ & 49.0 & 0.507 & 0.483 & 0.118 & 49.0 & 0.598 & 0.787 & 0.099 & 990608.2 \\
\hline $19195-1423^{a}$ & -75.0 & 1.857 & 4.784 & 0.197 & -75.2 & 1.350 & 4.776 & 0.147 & 990608.2 \\
\hline
\end{tabular}

Notes to Table 2:

${ }^{a}$ : These stars do not satisfy our selection criteria, and excluded from the following discussions.

comparison with the whole IRAS point sources. There are 6353 stars in the IRAS PSC which satisfy our color selection criteria and have $f_{12}>3 \mathrm{Jy}$. Among them, 1080 have $C_{12}$ larger than $0.0(17 \%)$. This may be interpreted as an indication that there are only a few massive AGB stars in the present high galactic latitude sample (Kwok 1990).

\subsection{Spatial distribution}

We used the following equation to calculate distances,

$D_{\mathrm{L}}=\sqrt{L /\left(4 \pi F_{\mathrm{bol}}\right)}$ where $L$ is the luminosity of the star. The bolometric flux $F_{\text {bol }}$ is calculated from the IRAS $12 \mu \mathrm{m}$ flux $f_{12}$ and bolometric correction factor $B C$,

$$
F_{\text {bol }}\left[\mathrm{W} / \mathrm{m}^{2}\right]=2.49 \times 10^{-13} \times B C \times f_{12}[\mathrm{Jy}] .
$$

For O-rich stars, $B C$ can be calculated by (van der Veen \& Breukers 1989)

$B C=0.7+2.9 \mathrm{e}^{-7.5 \times C_{12}}+0.9 \mathrm{e}^{1.75 \times C_{12}}$.

In Eq. (3), $C_{12}$ is the IRAS 12 and $25 \mu \mathrm{m}$ flux density ratio, defined in Sect. 2. 
Table 3. List of $\mathrm{SiO}$ maser non-detections.

\begin{tabular}{|c|c|c|c|c|c|c|c|}
\hline IRAS Name & $\begin{array}{c}J=1-0, v=1 \\
\mathrm{rms} \\
(\mathrm{K})\end{array}$ & $\begin{array}{c}J=1-0, v=2 \\
\mathrm{rms} \\
(\mathrm{K})\end{array}$ & $\begin{array}{c}\text { Observation } \\
\text { date } \\
\text { (yymmdd.d) }\end{array}$ & IRAS Name & $\begin{array}{c}J=1-0, v=1 \\
\text { rms } \\
(\mathrm{K})\end{array}$ & $\begin{array}{c}J=1-0, v=2 \\
\mathrm{rms} \\
(\mathrm{K})\end{array}$ & $\begin{array}{c}\text { Observation } \\
\text { date } \\
\text { (yymmdd.d) }\end{array}$ \\
\hline $08020+6637$ & 0.054 & 0.045 & 000608.5 & $08134+4017$ & 0.047 & 0.044 & 000411.8 \\
\hline $08258+6237$ & 0.051 & 0.045 & 000411.9 & $08430+3705$ & 0.050 & 0.045 & 000608.5 \\
\hline $09005+0824$ & 0.056 & 0.050 & 000409.8 & $09276+4454^{b}$ & 0.050 & 0.048 & 000526.7 \\
\hline 09343-0536 & 0.055 & 0.052 & 000409.8 & $09406+5359$ & 0.043 & 0.045 & 000410.8 \\
\hline 09439-0547 & 0.054 & 0.054 & 000526.6 & $10196+2545$ & 0.066 & 0.064 & 000410.8 \\
\hline $10353-1145$ & 0.065 & 0.057 & 000608.6 & $10457+3633$ & 0.045 & 0.044 & 000526.7 \\
\hline $10594-0723$ & 0.057 & 0.053 & 000409.9 & $11193-2443$ & 0.085 & 0.083 & 000608.7 \\
\hline $11252+1525$ & 0.054 & 0.049 & 000409.9 & $11269-1839$ & 0.060 & 0.057 & 000608.7 \\
\hline $11443+5830$ & 0.049 & 0.044 & 000411.9 & $11445+4344$ & 0.072 & 0.072 & 000410.8 \\
\hline $11472-0800^{b}$ & 0.051 & 0.050 & 000525.7 & $12020+0254$ & 0.056 & 0.052 & 000409.9 \\
\hline $12219-0445$ & 0.066 & 0.061 & 000608.6 & $12254+1904$ & 0.044 & 0.047 & 990607.8 \\
\hline $12323-2033$ & 0.065 & 0.055 & 000412.9 & $12326-2017$ & 0.070 & 0.066 & 000608.7 \\
\hline $12462+0738$ & 0.043 & 0.045 & 990607.8 & $12564-3125$ & 0.086 & 0.090 & 000609.9 \\
\hline $13006-2850$ & 0.065 & 0.071 & 990607.9 & $13053-3208$ & 0.081 & 0.070 & 000412.9 \\
\hline $13245-2446$ & 0.078 & 0.070 & 000412.9 & $13252-0254$ & 0.063 & 0.058 & 000409.9 \\
\hline $13326-2207$ & 0.057 & 0.068 & 000413.9 & $13367-3031$ & 0.072 & 0.074 & 000518.9 \\
\hline $13562-1342$ & 0.074 & 0.071 & 000519.0 & $13573+2801$ & 0.057 & 0.069 & 000413.9 \\
\hline $13582+3806$ & 0.192 & 0.187 & 000410.9 & $13594-0522$ & 0.049 & 0.047 & 000410.0 \\
\hline 14021-1909 & 0.041 & 0.040 & 990608.9 & $14142-1612$ & 0.055 & 0.052 & 000609.9 \\
\hline $14257-0530$ & 0.048 & - & 990610.9 & $14382-2504$ & 0.064 & 0.068 & 000609.9 \\
\hline $14436-0703$ & 0.079 & 0.078 & 000409.9 & $14534-0359$ & 0.060 & 0.060 & 000609.0 \\
\hline $15106-1532$ & 0.037 & 0.034 & 990607.9 & $15114-0142$ & 0.053 & 0.053 & 000410.0 \\
\hline $15194-1829$ & 0.057 & 0.058 & 000410.0 & $15223-0203$ & 0.058 & 0.055 & 980513.9 \\
\hline $15250+2952$ & 0.067 & 0.062 & 000608.8 & $15334+2555$ & 0.054 & 0.064 & 000413.9 \\
\hline $15367+1044$ & 0.051 & 0.046 & 000609.1 & $15415+0232$ & 0.033 & 0.032 & 000604.0 \\
\hline $15517-1043$ & 0.090 & 0.106 & 000413.9 & $15522-0749$ & 0.057 & 0.058 & 000603.9 \\
\hline $15540+0910$ & 0.057 & 0.042 & 980512.1 & $16211+3057$ & 0.067 & 0.081 & 000413.9 \\
\hline $16241-0230$ & 0.061 & 0.062 & 000411.0 & $16293+4900$ & 0.089 & 0.091 & 000608.8 \\
\hline $16330+0405$ & 0.081 & 0.070 & 000413.0 & $16400+3301$ & 0.051 & 0.043 & 000608.8 \\
\hline $16418+5435$ & 0.066 & 0.061 & 000608.9 & $16418+5459$ & 0.039 & 0.037 & 000604.1 \\
\hline $17313+7033$ & 0.054 & 0.050 & 000608.6 & $17343+5026$ & 0.061 & 0.060 & 000411.9 \\
\hline $17343+7601$ & 0.076 & 0.066 & 000608.6 & $17361+5746$ & 0.062 & 0.058 & 000608.9 \\
\hline $17403+6234$ & 0.064 & 0.053 & 000608.9 & $11331-1418^{a}$ & 0.126 & - & 990610.9 \\
\hline $17038-2033^{a}$ & 0.112 & 0.092 & 980512.1 & $17093-1633^{a}$ & 0.135 & 0.118 & 980525.9 \\
\hline $17221-1706^{a}$ & 0.084 & 0.071 & 980606.9 & $19083-0017^{a}$ & 0.053 & 0.059 & 990608.2 \\
\hline $19094+0006^{a}$ & 0.077 & 0.087 & 990608.2 & $19386+0155^{a}$ & 0.040 & 0.036 & 990611.2 \\
\hline $19438+0933^{a}$ & 0.037 & 0.031 & 990609.2 & $19457+0832^{a}$ & 0.037 & 0.034 & 990609.2 \\
\hline $19583-0730^{a}$ & 0.039 & 0.032 & 990611.2 & & & & \\
\hline
\end{tabular}

Notes to Table 3:

${ }^{a}$ These stars do not satisfy our selection criteria, and excluded from the following discussions.

${ }^{b}$ T. Fujii et al. in prep. (private communication).

The luminosity of each star, $L$ is calculated by using the period-bolometric magnitude relation for oxygen-rich Mira type variables (Van Leeuwen et al. 1997),

$M_{\mathrm{bol}}=-3.00 \log P+2.88$

and by adopting $M_{\odot \text { bol }}=4.72$ (Zombeck 1982), where $M_{\text {bol }}$ and $M_{\odot \text { bol }}$ are the absolute bolometric magnitude of each star and that of the Sun respectively. Pulsation periods have been found for 36 stars in the General Catalog of Variable Stars (GCVS) (Kholopov et al. 1992) and these are listed in Table 1. Their luminosities are calculated from the above equation. The median of the known periods is about 300 days. According to the above relation, a Mira type variable with the period of around 300 days would 


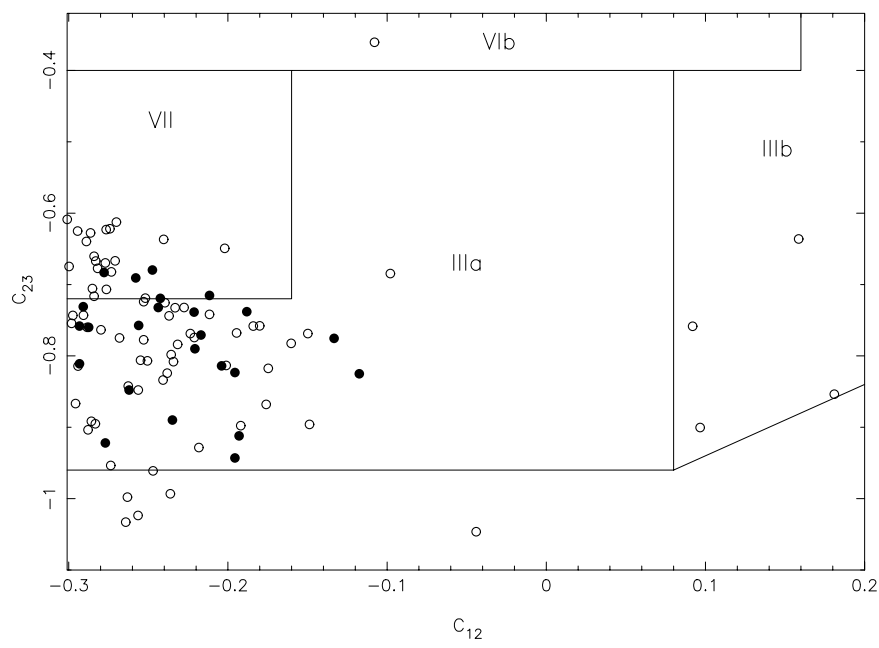

Fig. 2. IRAS two-color diagram of the present sample. The filled and open circles represent $\mathrm{SiO}$ detections and nondetections, respectively. Van der Veen \& Habing (1988) studied IRAS sources based on a two-color diagram which they divided into 10 regions, and these are also included.

have a luminosity of about $5000 L_{\odot}$. Thus, for the remaining 61 stars, we assumed that they all have the luminosity of $5000 L_{\odot}$. Distances derived are tabulated in Table 1 .

Having deduced the distance to the individual stars, we can then calculate the position of the stars in the heliocentric cartesian coordinates and look at their Galactic distribution, as shown in Fig. 3. There are more stars in the hemisphere towards the Galactic center (68 stars) than the anti-center ( 29 stars). To some extent, this trend comes from the effect that the number density of the star is falling with the Galactocentric radius. Among the $\mathrm{SiO}$ detected 24 stars, 18 are in the hemisphere towards the Galactic center and 6 are in the anti-center (see Fig. 3 middle and bottom). Thus the detection rate is $26 \%$ for the inner hemisphere and $20 \%$ for the outer one, respectively. However, there seems no such asymmetry in the $y z$ plane (see Fig. 3 top). The difference in two proportions test showed that this asymmetry in detection rate is meaningful with the $72 \%$ confidence level, unfortunately within the statistical error.

The trend for the lower detection rate in the outer Galactic disk could be explained as the number ratio of carbon to oxygen-rich stars increases for larger Galactic radii (Jura 1993). Jura (1992c) found the remarkable increase of the fraction of carbon-rich red giants in the outer Milky Way. Jiang et al. (1999) concluded that the decreasing sequence of $\mathrm{SiO}$ maser population with the galactocentric distance is possibly as a consequence of a variation in the proportion of oxygen-rich variable stars and the galactic metallicity gradient. A decrease in metallicity as a function of Galactic radius has been suggested by Shaver et al. (1983) and the relationship between metallicity and the ratio of carbon to oxygen-rich stars has been reported for external galaxies (Iben \& Renzini 1983; Richer 1989).
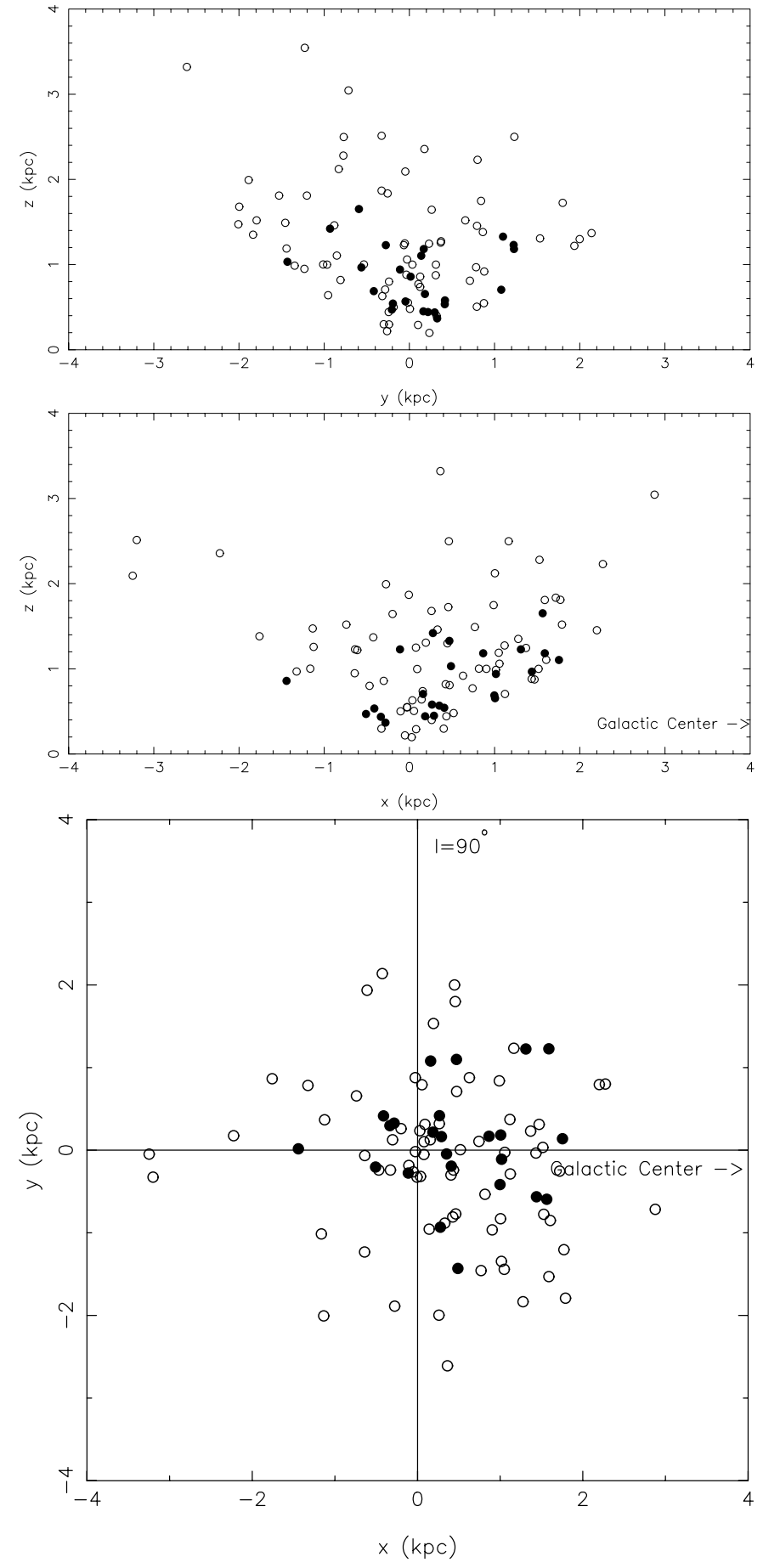

Fig. 3. The spatial distribution of stars in the heliocentric cartesian coordinates. The coordinates, $x, y$ and $z$ increase toward the Galactic center, anti Galactic rotation, and the North Galactic Pole, respectively. The filled circles represent SiO detections, and the open circles non-detections.

Figure 4 is a histogram of the distance from the Galactic plane $(z)$ of the present sample. The hatched area shows the number of $\mathrm{SiO}$ maser detections in each bin. There are no $\mathrm{SiO}$ detections above $1.8 \mathrm{kpc}$ from the Galactic plane. This may be an artifact of the distance estimation. If this is real however, it could be explained by 


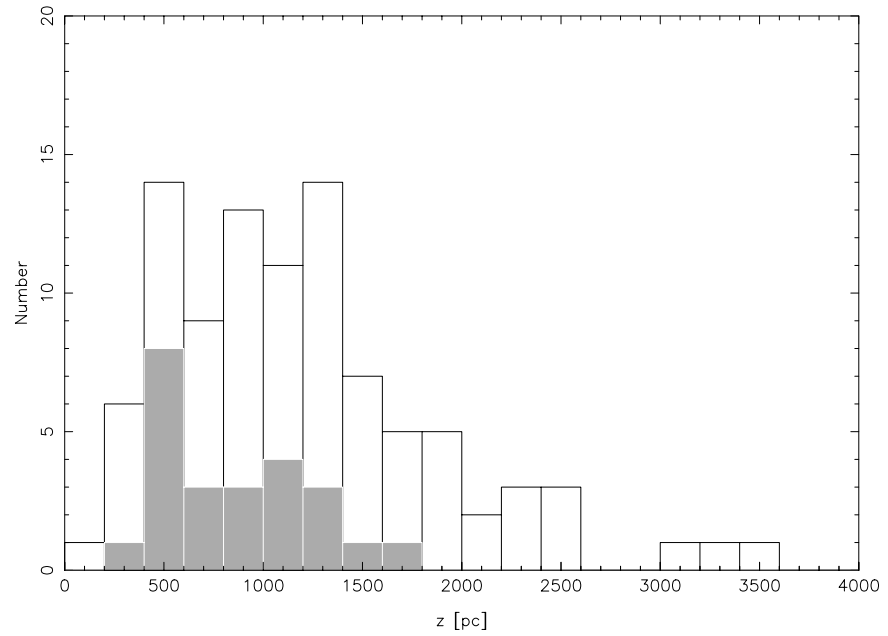

Fig. 4. Histogram of the Galactic height. The hatched area shows $\mathrm{SiO}$ maser detections, and the open area non-detections.

the metallicity gradient with the Galactic height. Norris \& Green (1989) observed a well-defined vertical composition gradient, given formally by $[\mathrm{Fe} / \mathrm{H}]=-0.19 z-0.16$ ( $z$ in $\mathrm{kpc}$ ). The lower metallicity away from the Galactic plane could lead to an increasing number of O-rich stars that turn into C-rich stars easily (Iben \& Renzini 1983).

\subsection{Galactic vertical kinematics}

To investigate the Galactic vertical kinematics, the sample is limited to stars with distance, proper motions and l.o.s. velocities. Distances were derived from the method discussed in the Sect. 4.3. Proper motions were taken from the Tycho-2 catalog (Høg et al. 2000a, 2000b) and stellar identifications were made by matching IRAS positions and catalog positions when they differ less than $10 \mathrm{~s}$ of arc. High precision l.o.s velocities were derived for 24 stars from $\mathrm{SiO}$ maser observations and for the remaining 73 stars, literature was searched. These data are listed in Table 4.

The distances, proper motions, and l.o.s. velocities were converted into the space velocity components in the Galactocentric cylindrical coordinates. The azimuthal rotational component in the plane relative to a non-rotating point at the Solar position $V_{\theta}$ was calculated. The $V_{\theta}$ has been corrected for the peculiar motion of the Sun $\left(u_{\odot}=8.1, v_{\odot}=7.4, w_{\odot}=6.4\right) \mathrm{km} \mathrm{s}^{-1}$ (Ratnatunga \& Arthur 1997) and $222 \mathrm{kms}^{-1}$ (Kerr \& Lynden-Bell 1986) as the azimuthal velocity of the LSR. A value of the distance to the Galactic center from the Sun of $8.5 \mathrm{kpc}$ was adopted. Then we calculated the angular velocities $(\omega)$ around the Galactic center by dividing $V_{\theta}$ by Galactocentric distance of each star.

In Fig. 5, we plot angular velocity versus Galactic height. If the Milky Way has a discrete thick disk, there should be an extra branch of objects with a different $\omega-z$ relation. Because there are some uncertainties in the
Table 4. Kinematical data.

\begin{tabular}{ccrrrr}
\hline \hline $\begin{array}{c}\text { IRAS } \\
\text { name }\end{array}$ & $\begin{array}{c}\text { Tycho-2 } \\
\text { ID }\end{array}$ & $\begin{array}{c}V_{\text {los }} \\
\left(\mathrm{km} \mathrm{s}^{-1}\right)\end{array}$ & $\begin{array}{c}R_{\mathrm{V}} \\
\mu_{\alpha} \\
(\mathrm{mas} / \mathrm{yr})\end{array}$ \\
\hline $09069+2527$ & $1954-01300-1$ & 49.0 & 2 & -15.70 & -11.80 \\
$10353-1145$ & $5495-00421-1$ & 49.0 & 3 & -52.70 & -9.90 \\
$11125+7524$ & $4549-01867-1$ & -50.0 & 1 & -16.30 & -13.80 \\
$11252+1525$ & $1437-01509-1$ & 6.0 & 3 & -23.00 & 10.40 \\
$13252-0254$ & $4962-00612-1$ & 33.0 & 2 & 5.50 & -4.20 \\
$14086-2839$ & $6742-00806-1$ & 2.0 & 2 & -10.20 & -32.30 \\
$14247+0454$ & $0321-00490-1$ & -26.0 & 2 & -6.70 & 1.90 \\
$14371+3245$ & $2557-00818-1$ & -3.3 & 2 & 15.20 & -5.70 \\
$15090-0549$ & $5013-00562-1$ & 17.4 & 1 & -9.00 & -18.60 \\
$15114-0142$ & $5005-00784-1$ & -59.0 & 2 & -31.60 & -9.60 \\
$15193+3132$ & $2563-01338-1$ & -1.0 & 2 & -9.70 & -15.90 \\
$15334+2555$ & $2029-01030-1$ & -27.0 & 2 & -7.40 & -10.80 \\
$15566+3609$ & $2578-00824-1$ & -50.2 & 1 & -51.60 & 0.30 \\
$16211+3057$ & $2580-00057-1$ & 28.0 & 3 & -17.30 & 6.10 \\
$16418+5459$ & $3879-01661-1$ & 6.0 & 2 & 3.30 & -32.30 \\
$16560+2252$ & $2059-00770-1$ & -2.8 & 1 & 3.60 & -5.20 \\
\hline
\end{tabular}

Notes to Table 4 :

ID: Tycho-2 ID number.

$V_{\text {los }}$ : Line of sight velocity.

$R_{\mathrm{V}}$ : Reference of the l.o.s. velocity.

1: This study.

2: General Catalogue of Stellar Radial Velocities (Wilson 1953).

3: The kinematics of semiregular red variables in the solar neighborhood (Feast et al. 1972).

$\mu_{\alpha}, \mu_{\delta}$ : Proper motion in RA and DEC.

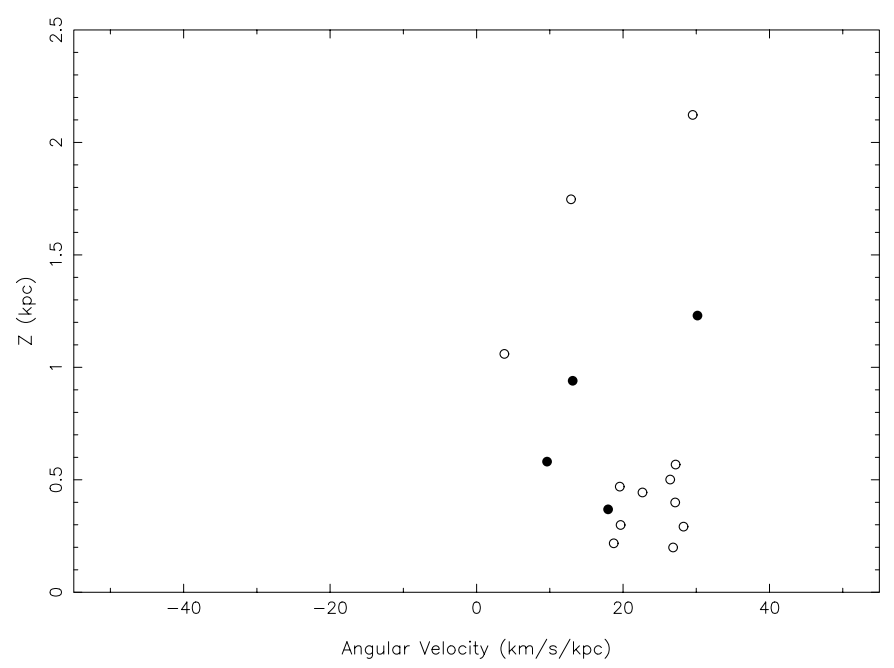

Fig. 5. The variation of angular velocity with the Galactic height. The filled circles indicate the stars of their l.o.s. velocities were determined by this study, and the open circles, data taken from the literature.

distance estimation and our subsample may not deep enough to contain much of thick disk population, the existence of some extra streams is not clear. 


\section{Conclusions}

We have observed color-selected IRAS sources in the North Galactic Cap, in the $\mathrm{SiO} J=1-0 v=1$ and 2 transitions, leading to 24 detections, of which 17 were new detections. The detection rate (about 25\%) was quite low. This is possibly a consequence of a variation in the Galactic metallicity and hence the decreasing proportion of oxygen-rich long period variables that are most likely to emit $\mathrm{SiO}$ masers. No $\mathrm{SiO}$ masers were found from the stars with the Galactic height larger than $1.8 \mathrm{kpc}$ in the present survey. We do not find any clear evidence for the existence of the extra branch in the $\omega-z$ plane.

Acknowledgements. It is a pleasure to thank Dr. H. Mito for useful and helpful discussions. This research has made use of the SIMBAD database operated at CDS, Strasbourg, France and partially based on data from the ESA Hipparcos astrometry satellite.

\section{References}

Benson, P. J., Little-Marenin, I. R., Woods, T. C., et al. 1990, ApJS, 74, 911

Cohen, R. J. 1989, Rep. Prog. Phys., 52, 881

Deguchi, S., \& Iguchi, T. 1976, PASJ, 28, 307

ESA 1997, The Hipparcos and Tycho Catalogs, ESA, SP 1200

Feast, M. W., Woolley, R., \& Yilmaz, N. 1972, MNRAS, 158, 23

Kholopov, P. N., Samus, N. N., Durlevich, O. V., et al. 1992, General catalogue of variable stars, fourth edition (Bull. Inf. Centre Données Stellaires)

Hall, P. J., Allen, D. A., Troup, E. R., et al. 1990, MNRAS, 243,480

Høg, E., Fabricius, C., Makarov, V. V., et al. 2000a, A\&A, 357, 367

Høg, E., Fabricius, C., Makarov, V. V., et al. 2000b, A\&A, 355, L27

Iben, I., \& Renzini, A. 1983, ARA\&A, 21, 271

IRAS Science Team 1988, IRAS Point Source Catalog, Version 2
Izumiura, H., Deguchi, S., Hashimoto, O., et al. 1994, ApJ, 437,419

Izumiura, H., Catchpole, R., Deguchi, S., et al. 1995a, ApJS, 98,271

Izumiura, H., Deguchi, S., Hashimoto, O., et al. 1995b, ApJ, 453,837

Izumiura, H., Deguchi, S., Fujii, T., et al. 1999, ApJS, 125, 257

Jewell, P. R., Snyder, L. E., Walmsley, C. M., et al. 1991, A\&A, 242,211

Jiang, B. W., Deguchi, S., Izumiura, H., et al. 1995, PASJ, 47, 815

Jiang, B. W., Deguchi, S., Yamamura, I., et al. 1996, ApJS, 106,463

Jiang, B. W., Deguchi, S., \& Ramesh, B. 1999, PASJ, 51, 95

Jura, M., \& Kleinmann, S. G. 1992a, ApJS, 83, 329

Jura, M., \& Kleinmann, S. G. 1992b, ApJS, 79, 105

Jura, M. 1992c, A\&AR, 2, 227

Jura, M., \& Yamamoto, A. 1993, ApJ, 413, 298

Jura, M. 1994, Ap\&SS, 217, 101

Kerr, F. J., \& Lynden-Bell, D. 1986, MNRAS, 221, 1023

Kerschbaum, F., \& Hron, J. 1992, A\&A, 263, 97

Kwok, S. 1990, MNRAS, 244, 179

Lane, A. P., Johnston, K. J., Spencer, J. H., et al. 1987, ApJ, 323,756

Miyoshi, M., Matsumoto, K., Kameno, S., et al. 1994, Nature, 371,395

Nakada, Y., Onaka, T., Yamamura, I., et al. 1993, PASJ, 45, 179

Norris, J. E., \& Green, E. M. 1989, ApJ, 337, 272

Ratnatunga, K. U., \& Upgren, A. R. 1997, AJ, 476, 811

Richer, H. B. 1989, IAU Coll. 106 (Cambridge University Press), 35

Shaver, P. A., McGee, R. X., Newton, L. M., et al. 1983, MNRAS, 204, 53

van der Veen, W. E. C. J., \& Habing, H. J. 1988, A\&A, 194, 125

van der Veen, W. E. C. J., \& Breukers, R. J. L. H. 1989, A\&A, 213, 133

van Leeuwen F., Feast, M. W., Whitelock, P. A., \& Yudin, B. 1997, MNRAS, 287, 955

Whitelock, P., Menzies, J., Feast, M., et al. 1994, MNRAS, 267,711

Wilson, R. E. 1953, Carnegie Inst. Washington DC, Publ. 601 Zombeck, Martin, V. 1982 (Cambridge University Press), 102 